\title{
Replacing overt verbal and gestural prompts with unobtrusive covert tactile prompting for students with autism
}

\author{
Heather M. Anson and James T. Todd \\ Eastern Michigan University, Ypsilanti, Michigan \\ AND \\ Kimberley J. Cassaretto \\ Central Valley Autism Project, Inc., Modesto, California
}

\begin{abstract}
Verbal responses, gestures, and other physical stimuli are often used to prompt children to pay attention to their teacher, participate in group responding, and engage in independent activities in the classroom. Prompts can be intrusive and draw attention to the problem, however. In the present study, unobtrusive vibrating pagers were used to discreetly alert children to attend directly to the teacher or the ongoing activity, thus reducing the number of disruptions the children created in their classrooms. The children were then able to learn more effectively and with less interference to others in the vicinity. Specifically, 5 male children, between 4 and 7 years old, who attended a regular education preschool or regular education first-grade classroom, participated. An alternating baseline and treatment conditions design was used, in which periods of overt traditional prompting were alternated with periods of covert tactile and overt traditional prompting. The data showed that covert tactile prompting was successful in reducing the amount of overt traditional prompting that was needed for attention to a teacher, group responding, and engagement in independent activities.
\end{abstract}

Prompts are very common instructional techniques within many kinds of behavioral interventions (Davis, Bostow, \& Heimisson, 2007; Ebanks \& Fisher, 2003; Fisher, Kodak, \& Moore, 2007). Technically speaking, a prompt is an action taken by an instructor that is intended to help a student engage in an appropriate response (Alberto \& Troutman, 2006; Lovaas, 2003). For people with developmental disabilities, prompts are typically verbal, physical, or gestural (Alberto \& Troutman, 2006). A teacher working with a child who does not speak well might model a verbal response as a prompt, and then reinforce the correct imitation (Alberto \& Troutman, 2006; Gena, Couloura, \& Kymissis, 2005). A person might be guided manually through a task, or a teacher might point to a series of objects to be named (Leaf \& McEachin, 1999; Lovaas, 2003; Maurice, Green, \& Luce, 1996). Prompts are generally faded as the control of the behavior is transferred to the natural environment (Alberto \& Troutman, 2006; Maurice et al., 1996). Although prompts are useful instructional techniques within behavioral interventions (Wright, Gurman, \& CASP, 2001), their overt nature can be distracting and can draw attention to a student's off-task behaviors or to the fact that the student needs assistance that is not required by other children. If prompts could be made less obtrusive, behavioral treatments might be more effectively incorporated into standard classroom activities.
Taylor and Levin (1998) demonstrated the effectiveness of a covert tactile device used to prompt a student with autism to make verbal statements about play activities. Shabani et al. (2002) compared the same type of covert tactile prompting with overt traditional prompting in efforts to increase on-topic verbalizations and appropriate socialization with peers. Both studies demonstrated that a covert tactile prompt was successful as a less intrusive method of prompting for the participants. The authors of the present study were interested in applying this type of prompting to a different class of deficit behaviors. They accomplished this by using an inexpensive, easily obtainable, unobtrusive vibrating pager to prompt on-task behavior during activities that required attention to the teacher, group responding, and engagement in independent activities.

\section{METHOD}
Participants
Five children who were diagnosed with autism on the basis of the criteria outlined in the Diagnostic and Statistical Manual of Mental Disorders (American Psychiatric Association, 2000) participated in the present study. At the time of the study, all 5 participants had been receiving early intensive behavioral treatment (EIBT) program- ming for at least 1 year through Central Valley Autism Project, Inc. (CVAP), a Dr. Ivar Lovaas Research and Clinical Replication Site. Prior to entering treatment, each participant had been evaluated at

H. M. Anson, hanson@emich.edu 
the Early Autism Screening Clinic. These evaluations consisted of a screening using the Pervasive Developmental Disorders Screening Test-II (Siegel, 2001), the Checklist for Autism in Toddlers (BaronCohen et al., 1996), and analysis of other pertinent medical, developmental, and social family data (Lovaas, 2003). In order for children to qualify for services with CVAP's EIBT program, they must meet specific criteria. These include the following: (1) The child must have a diagnosis of autism; (2) programming must begin before the age of 48 months if the child is nonverbal, or 50 months if some functional language is present; (3) the child's IQ scores must be 40 or greater (Bayley, 2006; Wechsler, 2002); and (4) there must be an absence of other medical conditions. All 5 participants had met these criteria prior to entering treatment with CVAP.

Three of the children, "Cody," "Jimmy," and "Tad," were 7 years old and attended first-grade regular education classrooms. The other 2 children, "Christopher" and "Evan," were 5 and 4 years old, respectively, and were in regular education preschool classrooms. All 5 children were selected because they had previously demonstrated on-task behaviors, including paying attention to the teacher, responding in unison with a group, and engaging in appropriate independent activities. On the basis of CVAP clinical supervisors' anecdotal report and classroom data, however, all participants had experienced difficulty in performing these skills reliably in their regular classroom environments.

Definitions. Appropriate on-task classroom behavior was defined and targeted for three different areas: (1) paying attention to the teacher (ATT), (2) group responding (GR), and (3) engaging in independent activities (EIA). ATT was defined as looking at the teacher or at relevant stimuli (e.g., a book or the chalkboard) while the teacher is talking. GR was defined as answering questions in unison with the class, singing songs with the class, or engaging in body movements along with the class. EIA was defined as working on an assigned project after the teacher has given instructions to begin the project, and continuing the project to completion.

\section{Materials}

A vibrating pager, a modified wireless door chime from Radio Shack (No. 63-872A), was used to supply the covert tactile prompt. The pager was activated by a handheld remote control, and it vibrated for approximately $2 \mathrm{sec}$ when activated. The delay between the activation of the remote control and the vibration of the pager was less than $1 \mathrm{sec}$. The pager was clipped to each participant's clothing and concealed from the view of others.

\section{Setting}

The initial training phase took place in the home of each participant in order to minimize the distractions from the classroom environment, and to decrease interference with the participants' participation in their typical classroom activities. Each child's home contained a room that was dedicated to one-on-one intervention with the child. This room, approximately $4 \times 4 \mathrm{~m}$, contained a childsized table and chairs, programming materials, and a variety of age-appropriate toys. The baseline and treatment phases occurred in the participants' regular education classrooms. The regular education preschool classrooms were typical of preschool classrooms in that they consisted of a large open area of approximately $8 \times 9 \mathrm{~m}$, which contained a "circle time" area, an arts-and-crafts table (which doubled as a snack table), and a variety of age-appropriate toys and activities with adequate space for engaging in typical play activities. The regular education first-grade classrooms were also typical, containing circle time areas, individual desks for each of 20 children, a large chalkboard, and a variety of academic activities.

\section{Procedure}

The experiment consisted of (1) a training phase to teach each participant how to respond to the covert tactile stimulus, and (2) a total of four alternating baseline and treatment conditions during which the covert tactile prompt's effectiveness in replacing verbal and gestural prompts was assessed. The training phase was conducted at home, concurrent with the first baseline condition at school. The tutors who conducted the training phase in the home and recorded the data at the schools were undergraduate and graduate students who were employed by CVAP. These tutors were very experienced in working with children with autism and in recording data. The tutors were trained to record data for the targeted appropriate classroom behaviors. As part of the training, they were given a copy of the operational definition for each type of targeted classroom behavior, and they were instructed in how to record data for these behaviors. They also participated in practice data collection sessions in order to ensure that they were trained to implement the prompt and to record the data adequately. Interrater reliability showed $90 \%-100 \%$ agreement during the training sessions.

During the training phase, each participant was taught by the tutors to respond appropriately to the covert tactile prompt during mock school activities in the home. Training sessions occurred once a day in 15- to 30-min sessions. Each participant was told, "This pager is to help you remember how to be a good student at school, but first we are going to practice at home." The participants were told that when they felt the vibration from the covert tactile prompt, they should respond with the appropriate school behavior. Participants first learned to respond appropriately to the covert tactile prompt when it was paired with a verbal prompt. Each participant was provided with contextual cues that identified the activity to engage in. These contextual cues were similar to the cues that were present in the school setting. For instance, when a participant engaged in inappropriate school behaviors, the covert tactile prompt was implemented and was paired with a verbal prompt. As a participant became successful in responding to both verbal and covert tactile prompting, the verbal prompt was systematically faded until it was no longer present. All participants were responding successfully to the covert tactile prompt after three to seven training sessions.

Each experimental condition lasted for 3 to 7 days. Condition lengths varied for each child and in each condition in order to ensure stability before a change in conditions. The different condition lengths created a design analogous to a multiple-baseline-acrossbehaviors design. The number of times that each child showed appropriate ATT, GR, or EIA was recorded during three 10-min wholeinterval recording periods during the day. Each of these observation periods was divided into twenty 30 -sec segments. During each of the two baseline conditions, the levels of appropriate classroom behavior were assessed when the participants received accustomed levels of verbal and gestural prompts. Verbal prompts consisted of instructions to the students such as "look at the teacher" or "do your work." Gestural prompts were physical movements such as pointing to the teacher or tapping the student's work to reestablish attention or task engagement. During each of the two treatment conditions, a covert tactile prompt from the vibrating pager was activated when the shadow tutor deemed that prompting was necessary, while behavior was measured as in the baseline conditions. For the ATT condition, a 3- to 5-sec break from looking at the teacher was allowed for appropriate off-task behavior (e.g., picking up a pencil or tying a shoelace) during each 30-sec interval. For the GR condition, a 3- to 5 -sec break in participation was allowed during each 30 -sec interval for appropriate off-task behavior (e.g., observing the motions to a new song). For the EIA condition, a delay of 5-15 sec (up to $15 \mathrm{sec}$ for preschoolers, up to $7 \mathrm{sec}$ for first graders) before starting the project itself was considered acceptable if the participants were doing something directly relevant to the project. After engagement had begun, each participant was allowed $5 \mathrm{sec}$ during each 30 -sec interval to engage in appropriate off-task behaviors (e.g., picking up a pencil, tying a shoelace, getting other materials out, or asking questions) without being considered off-task. If the covert tactile prompt was activated but the child did not respond appropriately within 2 to $3 \mathrm{sec}$, a verbal or gestural prompt was used as in the baseline phases. The experimenters thus hoped to determine whether there was a reduction in the number of verbal and gestural prompts that were required to maintain engagement by the child during the treatment conditions. 
Interrater reliability. Interrater reliability was measured during $25 \%$ of the observation intervals in each of the four phases. The second observer recorded exactly the same data on appropriate classroom behaviors as the primary observer did, and did not know when the covert tactile prompts were given. Interrater reliability ranged from $70 \%$ to $100 \%$, with an overall average of $96 \%$.

\section{RESULTS}

Figure 1 shows the percentage of intervals in which prompting for ATT, GR, and EIA was required to maintain their desired levels. The graphs also display the percentage of intervals in which the participant displayed appropriate responses and therefore required no prompting. These figures show that the unobtrusive covert tactile prompt was at least as effective for maintaining the desired level of each of the three target responses as overt traditional prompting was. In some of the children, patterns of covert tactile prompting presentation reduced the overall need for prompting.

Each participant showed different, but characteristic, responding to the prompt. Christopher, for instance, required the same number of prompts, or fewer, for each response class. His need for overt traditional prompts was slightly reduced during the covert tactile prompt conditions. Cody showed stabilized on-task behavior for ATT and EIA conditions and increases in appropriate behavior in GR during covert tactile prompt presentation. In addition, the number of overt traditional prompts he needed to maintain appropriate responses was reduced when the covert tactile prompt was used. Evan's need for prompts remained about the same in each condition, which demonstrated that the covert tactile prompts were at least not disruptive. Jimmy displayed a reduced need overall for overt traditional prompting, and he displayed more reliable appropriate behavior with the presence of the covert tactile prompt for each response class. Tad's appropriate behaviors remained relatively stable across conditions, and he needed slightly fewer overt traditional prompts after the introduction of the covert tactile prompt, which again demonstrated that there were no adverse behavioral reactions to the covert tactile prompt.

\section{DISCUSSION}

The results of the present study provide additional evidence supporting the utility of an unobtrusive covert tactile prompt for promoting on-task behavior in a standard classroom setting. Previous studies used a covert tactile prompt to improve on-topic verbalizations during play (Taylor \& Levin, 1998) and with peers (Shabani et al., 2002). The present study has extended these earlier findings to include improvements in new behavior classes (e.g., on-task behaviors, including ATT, GR, and EIA) and in other settings (e.g., routine classroom activities).

The goal of the present study was to reduce the need for overt traditional prompting by using covert tactile prompting instead. This goal was met. In almost all cases, less of the overt traditional prompting was required after the introduction of covert tactile prompting, and in most cases there was a reduction in the percentage of overt traditional prompting that was required in comparison with the baseline condition. It should be noted that the overall need for prompting did not change for most of the 5 participants. (Rather, the prompt type shifted from overt traditional to covert tactile.) Reducing overall prompting was not the goal of the present study, because prompting is a necessary component of training for these children and is faded over time on the basis of individual programming criteria.

Covert tactile prompting has several potential advantages over overt prompts. Tactile prompts can be delivered quickly and from anywhere near the child. Covert prompting does not require that the teacher disturb ongoing attention or disrupt activities to deliver the prompt. It is less obtrusive, drawing less attention from others to the off-task behavior of the child. Finally, covert tactile prompting reduces the possibility that instructions in the overt traditional prompt, expressed as an instruction or a gesture, might be misunderstood by the child.

Recent technological advances have expanded the options for tactile prompts. One possible alternative to the pager device that was used in the present study is a cell phone, set to vibrate mode and placed in the child's pocket. The cell phone could be activated by a call to the phone, using the speed-dial feature of another phone. Using cell phones as covert tactile prompts provides an additional advantage by creating an automatic record of the exact time that each prompt is delivered. The advent of small, low-cost, Bluetooth-based microcontrollers offers additional means by which covert prompts might be delivered, including multiple distinct prompts delivered by Bluetooth-enabled phones and small key-fob-type devices (Igoe, 2007). Future research will be required in order to explore the possibility of using other computerized devices to provide covert tactile prompts.

Additional research will be needed in order to fully examine the questions asked by the present study: To what extent can covert prompts replace overt traditional prompts? Do they maintain their function across time and settings? Are multiple covert prompts possible, and how might they be implemented? Would the relative ease of delivering covert prompts of this type increase their use by teachers, resulting in habituation to the prompts or prompt dependence? The manner by which covert prompts are faded is also critical (Alberto \& Troutman, 2006). Difficulties with fading might undermine any other advantages of the system.

There were two main limitations to the present study. First, data collection was based on a whole-interval recording procedure. Whole-interval recording can underestimate behavior (Alberto \& Troutman, 2006; Wright et al., 2001), which means that participants may have been on task more often than the data demonstrated. It will be important for future research to examine whether significant underestimation of on-task behavior occurred, and if so, how great it may have been. Second, the present study did not employ social validity measures (Codding, Feinberg, Dunn, \& Pace, 2005; Francisco \& Butterfoss, 2007; Schilling \& Schwartz, 2004) to assess how the tactile prompt might have affected the lives of the participants. Anecdotal reports from each of the participants' teachers, however, 


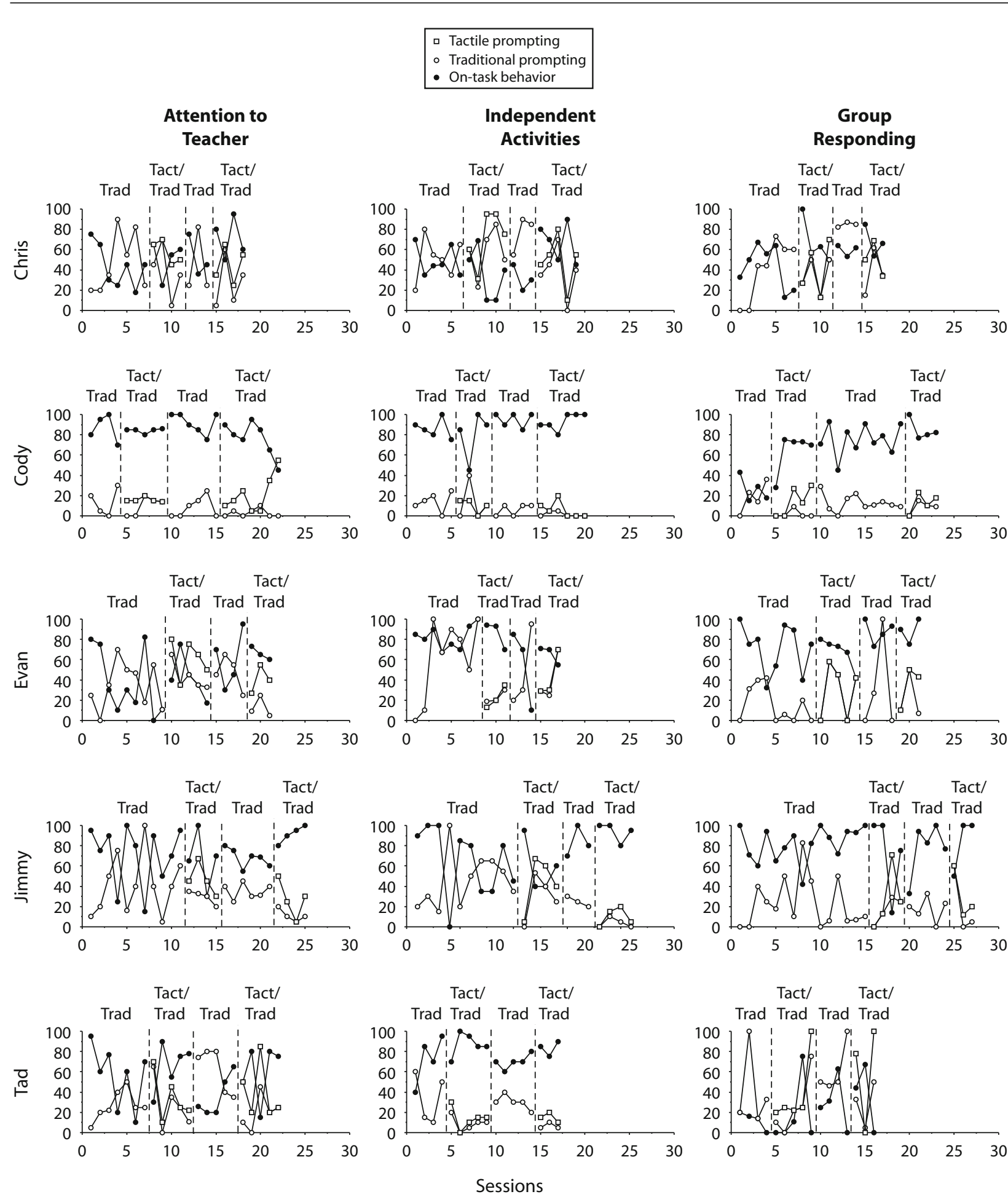

Figure 1. The percentages of 10-min recording intervals in which on-task behavior occurred (closed circles), verbal and gestural prompts were provided (open circles), and tactile prompts occurred (open squares). Trad and Traditional indicate conditions in which overt traditional verbal and gestural prompting was delivered. Tact indicates conditions in which covert tactile prompting occurred.

provided preliminary information that the tactile prompts were helpful in decreasing distractions in the classroom. Fewer classroom distractions resulted in the participants' appearing less impaired both to classroom teachers and to classmates. In addition, tutor reports indicated that the tactile-prompt device allowed staff to remain unobtrusive to the participants in their classrooms, thereby allowing the participants to be more independent. In some cases, par- 
ticipants indicated verbally that they preferred the covert tactile prompt over the overt traditional prompts. Finally, reports from CVAP indicated that tactile prompting continued to be used as the primary means of prompting in the classroom after the conclusion of this research project for 4 out of the 5 participants. Given this information, it will be important and useful for future research to formally assess the social validity of using covert tactile prompts.

\section{AUTHOR NOTE}

This study was a thesis presented to the faculty of California State University, Stanislaus, by H.M.A. in partial fulfillment of the requirements for an MS degree in Psychology. All data were collected at California State University, Stanislaus, and the Central Valley Autism Project. Portions of this work were presented as a poster at the annual meeting of the Behavior Analysis Association of Michigan, Ypsilanti, MI, February 2005, and at the annual meeting of the Association for Behavior Analysis, Chicago, IL, May 2005. H.M.A. acknowledges her thesis committee-Bruce E. Hesse, William F. Potter, and Gina M. Pallotta, Department of Psychology and Child Development, California State University, Stanislaus - for their assistance and advice in earlier versions of the manuscript. H.M.A. is now at Eastern Michigan University. Correspondence should be addressed to H. M. Anson, Department of Psychology, Eastern Michigan University, 537 Mark Jefferson, Ypsilanti, MI 48197 (e-mail: hanson@emich.edu).

\section{REFERENCES}

Alberto, P. A., \& Troutman, A. C. (2006). Applied behavior analysis for teachers (7th ed.). Upper Saddle River, NJ: Prentice Hall.

American Psychiatric Association (2000). Diagnostic and statistical manual of mental disorders (4th ed., text rev.). Washington, DC: Author.

Baron-Cohen, S., Cox, A., Baird, G., Swettenham, J., NightinGale, N., Morgan, K., ET AL. (1996). Psychological markers in the detection of autism in infancy in a large population. British Journal of Psychiatry, 168, 158-163.

Bayley, N. (2006). Bayley Scales of Infant and Toddler Development (3rd ed.). San Antonio, TX: Psychological Corporation.

Codding, R. S., Feinberg, A. B., Dunn, E. K., \& Pace, G. M. (2005). Effects of immediate performance feedback on implementation of behavior support plans. Journal of Applied Behavior Analysis, 38, 205-219.

Davis, D. R., Bostow, D. E., \& Heimisson, G. T. (2007). Strengthening scientific verbal behavior: An experimental comparison of progressively prompted and unprompted programmed instruction and prose tutorials. Journal of Applied Behavior Analysis, 40, 179-184.
EbANKs, M. E., \& Fisher, W. W. (2003). Altering the timing of academic prompts to treat destructive behavior maintained by escape. Journal of Applied Behavior Analysis, 36, 355-359.

Fisher, W. W., KodaK, T., \& Moore, J. W. (2007). Embedding an identity-matching task within a prompting hierarchy to facilitate acquisition of conditional discriminations in children with autism. Journal of Applied Behavior Analysis, 40, 489-499.

Francisco, V. T., \& Butterfoss, F. D. (2007). Social validation of goals, procedures, and effects in public health. Health Promotion Practice, 8, 128-133.

Gena, A., Couloura, S., \& Kymissis, E. (2005). Modifying the affective behavior of preschoolers with autism using in-vivo or video modeling and reinforcement contingencies. Journal of Autism \& Developmental Disorders, 35, 545-556.

IgOE, T. (2007). Making things talk: Practical methods for connecting physical objects. North Sebastopol, CA: Make Books.

LEAF, R., \& McEACHIN, J. (1999). A work in progress: Behavior management strategies and a curriculum for intensive behavioral treatment of autism. New York: DRL Books.

LovaAs, O. I. (2003). Teaching individuals with developmental delays. Basic intervention techniques. Austin, TX: Pro-Ed.

Maurice, C., Green, G., \& Luce, S. C. (1996). Behavioral intervention for young children with autism. Austin, TX: Pro-Ed.

Schilling, D. L., \& Schwartz, I. S. (2004). Alternative seating for young children with autism spectrum disorder: Effects on classroom behavior. Journal of Autism \& Developmental Disorders, 34, 423-432.

Shabani, D. B., Katz, R. C., Wilder, D. A., Beauchamp, K., Taylor, C. R., \& FISCHER, K. J. (2002). Increasing social initiations in children with autism: Effects of a tactile prompt. Journal of Applied Behavior Analysis, 35, 79-83.

SiEgeL, B. (2001). Pervasive Developmental Disorders Screening Test-II. San Antonio, TX: Pearson Education.

TAYlOR, B. A., \& LEVIN, L. (1998). Teaching a student with autism to make verbal initiations: Effects of a tactile prompt. Journal of Applied Behavior Analysis, 31, 651-654.

Wechsler, D. (2002). Wechsler Preschool and Primary Scale of Intelligence (3rd ed.). San Antonio, TX: Psychological Corporation.

Wright, D. B., Gurman, H. B., \& the California Association of School Psychologists/Diagnostic Center, Southern CaliforNia Positive Intervention Task Force (2001). Positive intervention for serious behavior problems: Best practices in implementing the Hughes Bill (A.B. 2586) and the positive behavioral intervention regulations (Rev. ed.). Sacramento: California Department of Education.

(Manuscript received November 1, 2007; revision accepted for publication March 27, 2008.) 